ARTICLE

\title{
Rough and smooth variants of Mycobacterium abscessus are differentially controlled by host immunity during chronic infection of adult zebrafish
}

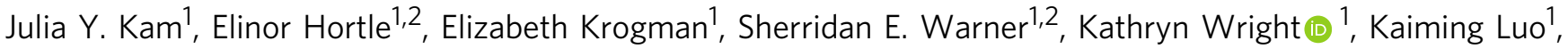
Tina Cheng (1) 1, Pradeep Manuneedhi Cholan', Kazu Kikuchi3,4, James A. Triccas (1) 2, Warwick J. Britton (1) 1,5, Matt D. Johansen (D) ${ }^{6}$, Laurent Kremer (D) ${ }^{6,7}$ \& Stefan H. Oehlers (D) 1,2,8凶

Prevalence of Mycobacterium abscessus infections is increasing in patients with respiratory comorbidities. After initial colonisation, M. abscessus smooth colony (S) variants can undergo an irreversible genetic switch into highly inflammatory, rough colony $(R)$ variants, often associated with a decline in pulmonary function. Here, we use an adult zebrafish model of chronic infection with $\mathrm{R}$ and $\mathrm{S}$ variants to study $M$. abscessus pathogenesis in the context of fully functioning host immunity. We show that infection with an $\mathrm{R}$ variant causes an inflammatory immune response that drives necrotic granuloma formation through host TNF signalling, mediated by the tnfa, tnfr1 and tnfr 2 gene products. T cell-dependent immunity is stronger against the $R$ variant early in infection, and regulatory $T$ cells associate with $R$ variant granulomas and limit bacterial growth. In comparison, an $\mathrm{S}$ variant proliferates to high burdens but appears to be controlled by TNF-dependent innate immunity early during infection, resulting in delayed granuloma formation. Thus, our work demonstrates the applicability of adult zebrafish to model persistent $M$. abscessus infection, and illustrates differences in the immunopathogenesis induced by $\mathrm{R}$ and $\mathrm{S}$ variants during granulomatous infection.

\footnotetext{
${ }^{1}$ Tuberculosis Research Program at the Centenary Institute, The University of Sydney, Camperdown, NSW, Australia. ${ }^{2}$ The University of Sydney, Faculty of Medicine and Health \& Marie Bashir Institute, Camperdown, NSW, Australia. ${ }^{3}$ Developmental and Stem Cell Biology Division, Victor Chang Cardiac Research Institute, Darlinghurst, NSW, Australia. ${ }^{4}$ St. Vincent's Clinical School, University of New South Wales, Kensington, NSW, Australia. ${ }^{5}$ Department of Clinical Immunology, Royal Prince Alfred Hospital, Camperdown, NSW, Australia. ${ }^{6}$ Centre National de la Recherche Scientifique UMR 9004 , Institut de Recherche en Infectiologie de Montpellier (IRIM), Université de Montpellier, Montpellier, France. ${ }^{7}$ INSERM, IRIM, Montpellier, France. ${ }^{8}$ A*STAR Infectious

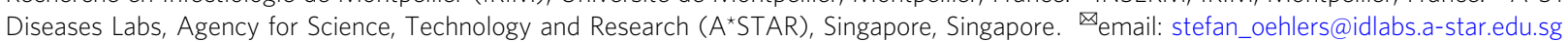


M ycobacterium abscessus is an increasingly recognized human pathogen responsible for a wide array of clinical manifestations including muco-cutaneous infections and disseminated or chronic pulmonary diseases ${ }^{1}$. The latter is mostly encountered in patients with underlying lung disorders, such as bronchiectasis or cystic fibrosis (CF). Irrespective of being a rapid-growing mycobacteria (RGM), $M$. abscessus displays many pathophysiological traits with slowgrowing mycobacteria (SGM), such as Mycobacterium tuberculosis. These include the capacity to persist silently within granulomatous structures and to produce pulmonary caseous lesions ${ }^{2,3}$. In addition, $M$. abscessus is notorious for being one of the mostdrug resistant mycobacterial species, characterized by a wide panel of acquired and innate drug resistance mechanisms against nearly all anti-tubercular drugs, as well as many different classes of antibiotics ${ }^{1,4}$. Consequently, this explains the complexity and duration of the treatments and the high level of therapeutic failure ${ }^{5}$.

M. abscessus exists either as smooth (S) or a rough (R) colony morphotype variants associated with distinct clinical outcomes ${ }^{6}$. Previous epidemiological studies have highlighted the association of the $\mathrm{R}$ variant that can persist for many years in the infected host, with a rapid decline in pulmonary function ${ }^{7-9}$. It is well established that these morphological differences between $\mathrm{S}$ and $\mathrm{R}$ variants are dependent on the presence or absence of surfaceexposed glycopeptidolipids (GPL), respectively, which are reduced or lost in $\mathrm{R}$ variants by transcriptional downregulation or loss of function mutations ${ }^{6,10-14}$. However, our knowledge of the pathophysiological characteristics and interactions between $\mathrm{R}$ or $\mathrm{S}$ variants with the host immune cells remains largely incomplete and is hampered by the lack of animal models that are permissive to persistent $M$. abscessus infection ${ }^{15}$.

Intravenous injection or aerosol administration of $M$. abscessus in immunocompetent $\mathrm{BALB} / \mathrm{c}$ mice fails to establish a persistent infection, typified by the rapid clearance of the bacilli from the liver, spleen and lungs within 4 weeks ${ }^{16}$. Immunosuppression is required to produce a progressive high level of infection with $M$. abscessus in mice, either genetically, as evident in nude, severe combined immunodeficiency (SCID), interferon-gamma (GKO) and granulocyte-macrophage colony-stimulating factor (GM-CSF) knock-out mice, or chemically by dexamethasone treatment ${ }^{17,18}$. Recently, an improved model of intratracheal agar bead inoculation has been adapted for $M$. abscessus causing a persistent infection for at least two months post implantation 17,19 .

The contribution of $\mathrm{B}$ and $\mathrm{T}$ cells in the control of $M$. abscessus infection has been studied in C57BL/6 mice with $\mathrm{Rag}^{-1-}, \mathrm{Cd} 3 \mathrm{e}^{-1-}$ and $\mu \mathrm{MT}^{-1-}$ knockouts $^{20}$. These studies indicated that infection control was primarily $\mathrm{T}$ cell-dependent in the spleen, and both $\mathrm{B}$ and $\mathrm{T}$ cell-dependent in the liver. In addition, IFN $\gamma$-receptor $\mathrm{KO}$ mice (ifngr $1^{-l-}$ ) were found to be significantly impaired in their control of $M$. abscessus both in the spleen and in the liver, as were $\mathrm{TNF}^{-1-}$ mice that had more pronounced and markedly dysregulated granulomas $^{20}$. Collectively, these findings highlight the central role of T cell immunity, IFN $\gamma$ and TNF for the control of M. abscessus in C57BL/6 mice, similarly to the control of $M$. tuberculosis infection.

In recent years, alternative non-mammalian models, such as Drosophila $^{21}$, Galleria larvae ${ }^{22}$, and zebrafish embryos ${ }^{15}$ have been developed to study the chronology and pathology of $M$. abscessus infection and for in vivo therapeutic assessment of drugs active against $M$. abscessus. In particular, zebrafish embryos have delivered important insights into the pathogenesis of $M$. abscessus and the participation of innate immunity in controlling infection ${ }^{10,23}$. The optical transparency of zebrafish embryos has been used to visualise the formation of large extracellular cords by the $\mathrm{R}$ form in vivo, representing a mechanism of immune subversion by preventing phagocytic destruction and highlighting the importance of mycobacterial virulence factors such as the dehydratase MAB_4780 and the MmpL $8_{\text {MAB }}$ lipid transporter ${ }^{10,24,25}$. Other studies in zebrafish embryos have demonstrated the contribution of host TNF signalling and IL8-mediated neutrophil recruitment for protective granulomatous immunity against $M$. $a_{\text {abcessus }}^{23}$, and the link between dysfunctional cystic fibrosis transmembrane conductance regulator (CFTR) and vulnerability to $M$. abscessus infection via phagocytic oxidative responses ${ }^{26}$.

Adult zebrafish models have been well-described for the study of mycobacterial pathogenesis by Mycobacterium marinum, used as a surrogate for the closely related M. tuberculosis, and the human pathogen Mycobacterium leprae ${ }^{27-30}$. Encompassing a fully functional immune system, previous studies in adult zebrafish with pathogenic mycobacteria, such as M. marinum, have unravelled the interplay between innate and adaptive immunity in mycobacterial granuloma formation and function.

Here, we show adult zebrafish are a useful host to analyse and compare the chronology of infection with M. abscessus $\mathrm{S}$ and $\mathrm{R}$ variants and to study the contribution of the $\mathrm{T}$ cell-mediated immunity in the granulomatous response in M. abscessus infection.

\section{Results}

Adult zebrafish can be chronically infected with M. abscessus. We infected adult zebrafish with approximately $10^{5} \mathrm{CFU}$ per animal with the rough (R) and smooth (S) variants of the reference strain CIP104536 ${ }^{\mathrm{T}}$, scaled for the smaller size of zebrafish from $10^{6}-10^{7}$ used in mouse intravenous infections ${ }^{16,17,20}$. To determine if $M$. abscessus produces a persistent infection in adult zebrafish, we performed CFU recovery on animals across 28 days of infection (Fig. 1A). Variation in the initial inoculum ranging from $10^{4}$ to $10^{6} \mathrm{CFU}$ did not appear to impact the course of infection burden with stable burden of the $\mathrm{R}$ variant within a 1-log window either side of the inoculation dose up to 28 days post infection (dpi) and progressive growth of the $S$ variant to approximately 1-log above the inoculation dose at $28 \mathrm{dpi}$ in each of three experiments.

Normalising burdens across three independent experiments per M. abscessus variant to perform statistical testing, we observed statistically significant increases in the proliferation of $M$. abscessus $\mathrm{S}$ compared to $\mathrm{R}$ variant at 7, 14, and 28 dpi (Fig. 1B). Furthermore, comparison of the Day 0 and Day 28 burdens demonstrated $M$. abscessus $\mathrm{R}$ burdens were statistically unchanged ( $P>0.99$, ANOVA) while $M$. abscessus $\mathrm{S}$ burdens increased by approximately 20 -fold across the 4 weeks $(P=0.024$, ANOVA).

Granuloma histopathology is accelerated during $M$. abscessus $\mathrm{R}$ infection compared to $\mathrm{S}$. We next performed histology on adult zebrafish infected with fluorescent $M$. abscessus $\mathrm{R}$ or S carrying tdTomato or Wasabi encoding plasmids ${ }^{10}$. From $10 \mathrm{dpi}$, we noted a heterogeneous mix of free bacteria around the peritoneal cavity or cellular granulomas diffuse foci of bacteria spread throughout host abdominal organ tissue, and necrotic granulomas with stereotypical host nuclei ringing around a central necrotic core containing M. abscessus in all animals (Fig. 2A).

We also observed the appearance of very large necrotic granulomas filled with fluorescent bacteria expressing tdTomato and necrotic debris measuring over $500 \mu \mathrm{m}$ in $M$. abscessus $\mathrm{R}$-infected animals from $14 \mathrm{dpi}$ onwards (Fig. 2A). These large granulomas were observed only occasionally and at a rate of no more than 1 per infected animal at 14 and 28 dpi $(n=2$ with single abscess, 9 without abscess). Three $M$. abscessus R-infected animals were maintained until $70 \mathrm{dpi}$ and appeared to be outwardly healthy. All three were found to have multiple necrotic granulomas containing fluorescent $M$. abscessus $\mathrm{R}$, demonstrating that persistent infection is possible in adult zebrafish, and two 

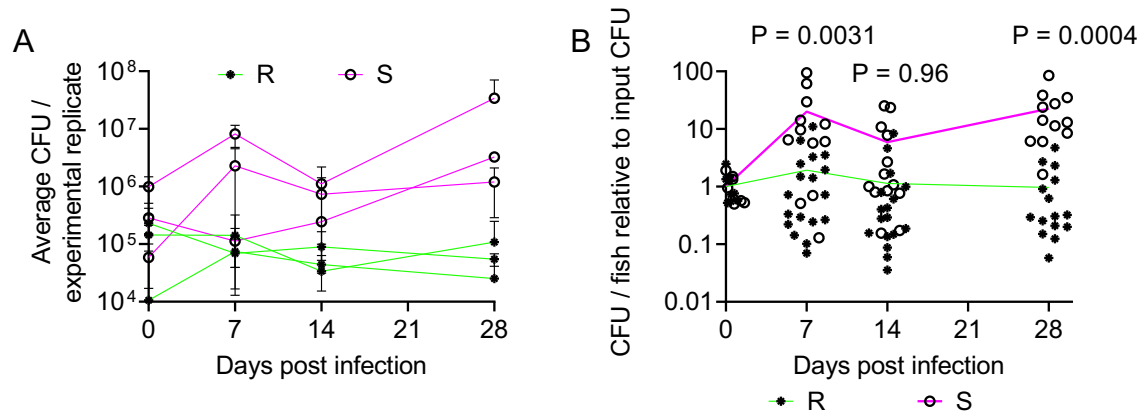

Fig. 1 M. abscessus establishes chronic infection in adult zebrafish. A Enumeration of CFUs from adult zebrafish infected with either the $R$ or the $S$ variant of $M$. abscessus. Each point represents a single experimental replicate with at least three animals per timepoint. Total $n$ per timepoint: $0 \mathrm{dpi} R=13 \mathrm{~S}=12 ; 7$ dpi $R=18 S=12 ; 14$ dpi $R=17 S=13 ; 28$ dpi $R=15 S=12$. B Relative CFUs recovered from adult zebrafish infected with either the $R$ or the $S$ variant of $M$. abscessus. Absolute CFU values were normalised to the inoculum CFU for each experimental replicate. Data are pooled from three replicates per $M$. abscessus variant. Total $n$ per timepoint: 0 dpi $R=13 \mathrm{~S}=12 ; 7$ dpi $R=18 \mathrm{~S}=12 ; 14 \mathrm{dpi} R=17 \mathrm{~S}=13 ; 28$ dpi $R=15 \mathrm{~S}=12$. Statistical tests by two-sided Student's $t$ test at each timepoint. Data are presented as mean values \pm SD. Source data are provided as a Source Data file.

were found to have necrotic granulomas measuring over $500 \mu \mathrm{m}$ suggesting an increase in the rate of large granuloma formation as infections progress (Supplemental Fig. 1). Granulomas in $M$. abscessus S-infected animals did not reach this size at the 14 and 28 dpi timepoints sampled ( $n=17$ at $14 \mathrm{dpi}, 16$ at $28 \mathrm{dpi}$ without abscess).

We next quantified the number of necrotic granulomas and "unorganised lesions", consisting of either diffuse foci of bacteria spread throughout host tissue or free bacteria around the peritoneal cavity. The proportion of necrotic granulomas in $M$. abscessus R-infected adult zebrafish increased between 10 and 14 dpi then remained similar between 14 and 28 dpi (Fig. 2B). $M$. abscessus $\mathrm{S}$ was observed to grow freely in mesenteric spaces and the rate of necrotic granulomas increased across all times with a statistically significant difference between 10 and 28 dpi (Fig. 2B). The proportion of necrotic granulomas was higher in $M$. abscessus R-infected than in M. abscessus S-infected animals at 14 dpi but similar at 10 and $28 \mathrm{dpi}$, suggesting granuloma formation is accelerated in $\mathrm{R}$ infections compared to $\mathrm{S}$ infections.

These patterns were recapitulated in our quantification of fluorescent bacterial burden in each type of lesion per animal. Significantly more $M$. abscessus $\mathrm{R}$ was observed within necrotic granulomas at 14 and $28 \mathrm{dpi}$ than at $10 \mathrm{dpi}$, and an increase in the proportion of $M$. abscessus $\mathrm{S}$ within necrotic granulomas was only observed at $28 \mathrm{dpi}$ compared to $10 \mathrm{dpi}$ (Fig. 2C). The proportion of $M$. abscessus $\mathrm{R}$ within necrotic granulomas was higher than the proportion of $M$. abscessus S within necrotic granulomas at $14 \mathrm{dpi}$ but similar at 10 and $28 \mathrm{dpi}$, again suggesting accelerated granuloma formation in $\mathrm{R}$ variant infections compared to $\mathrm{S}$.

Tumour necrosis factor-mediated immunity differentially controls infection by $\boldsymbol{M}$. abscessus variants. The cytokine TNF is essential for the granulomatous containment of M. abscessus in zebrafish embryos ${ }^{23}$. To visualize tnfa transcription, we next took advantage of the TgBAC(tnfa:GFP)pd1028 zebrafish line ${ }^{31}$, where GFP expression is driven by the tnfa promoter, to investigate if TNF expression is linked to granuloma formation. Expression of GFP was observed in adult zebrafish infected with either variant of $M$. abscessus. GFP was expressed by host cells in close contact with either M. abscessus R (Fig. 3A) or S (Fig. 3B).

To examine the role of host inflammatory signalling in the adult zebrafish- $M$. abscessus infection model, we first treated infected adults with the potent anti-inflammatory drug dexamethasone ${ }^{32}$. Dexamethasone treatment reduced tnfa promoter activity around M. abscessus lesions in TgBAC(tnfa:GFP)pd1028 zebrafish (Fig. 3C). Additionally, and as expected, dexamethasone treatment increased both $\mathrm{R}$ and $\mathrm{S}$ M. abscessus burdens (Fig. 3D), indicating that ablation of the inflammatory response led to uncontrolled bacterial expansion.

As dexamethasone acts broadly, we next specifically examined the role of the TNF-TNFR axis by knocking down expression of tnfa using a pooled gRNA CRISPR-Cas9 strategy. Comparison of WT and scrambled gRNA/Cas9-injected clutchmates indicated no effect of scrambled gRNA/Cas9-injection on M. abscessus R or $S$ burdens (Supplemental Fig. 2). The efficacy of knockdown was validated by reduced RT-qPCR detection of $\operatorname{tnfa}$ transcripts in CRISPR-Cas9 injected adults (Fig. 4A). Knockdown of tnfa reduced the burden of $\mathrm{R} M$. abscessus and increased the burden of S M. abscessus at $14 \mathrm{dpi}$ (Fig. 4B).

While tnfa-depleted animals formed necrotic granulomas at the same rate as control animals in response to $\mathrm{R} M$. abscessus infection (Fig. 4C), there was a sharply reduced proportion of M. abscessus $\mathrm{R}$ variant found within necrotic granulomas in tnfa-depleted animals (Fig. 4D). These parameters were unchanged in S M. abscessus infections suggesting the increased burden in S-infected tnfa knockdown animals was due to non-granulomatous mechanisms.

To further investigate the role of TNF signalling in our $M$. abscessus infection model, we next individually targeted the genes encoding the two zebrafish TNF receptors tnfr1 and tnfr2 for knockdown using CRISPR-Cas9 injections. Knockdown was confirmed by high resolution melt analysis of amplicons containing the first CRISPR target site demonstrating efficient editing of the loci (Fig. 4E). Knockdown of $t n f r 1$ or $t n f r 2$ again reduced the burden of $\mathrm{R} M$. abscessus and increased the burden of S M. abscessus at 14 dpi (Fig. 4F).

Necrotic granuloma formation was reduced in both $\operatorname{tnfr} 1$ and tnfr2 knockdown animals infected with $\mathrm{R} M$. abscessus, but unaffected in S M. abscessus-infected animals (Fig. 4G). The proportion of R M. abscessus, but not S M. abscessus, found within necrotic granulomas was also reduced in both tnfrl and tnfr2 knockdown animals (Fig. $4 \mathrm{H}$ ).

These observations demonstrate conservation of protective TNFmediated immunity against the $\mathrm{S}$ variant in adult infections and uncover a detrimental role for the TNF-TNFR axis in promoting $\mathrm{R}$ variant immunopathology through granuloma necrosis.

T cell-mediated immunity differentially controls infection by M. abscessus variants. Given the requirement for $\mathrm{T}$ cells to maintain granuloma structure in adult zebrafish $M$. marinum infection $^{29}$, we next asked if there was $\mathrm{T}$ cell involvement around M. abscessus granulomas using TgBAC(lck:EGFP) ${ }^{v c c 4}$ zebrafish $^{33}$. We observed $\mathrm{T}$ cell association and penetration throughout cellular 


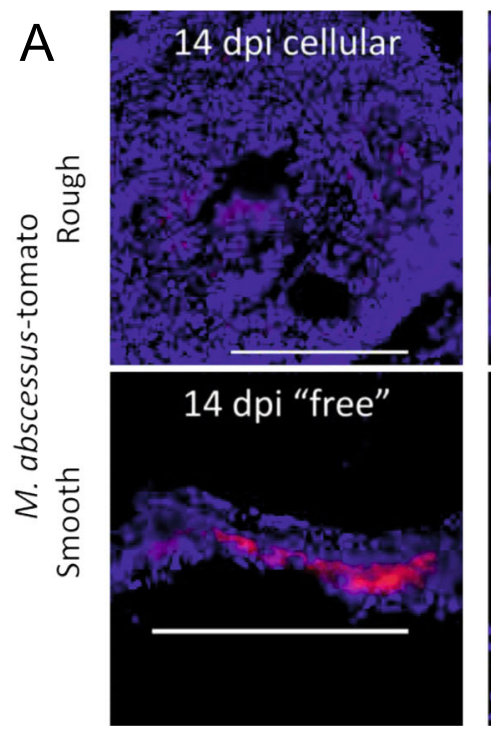

B

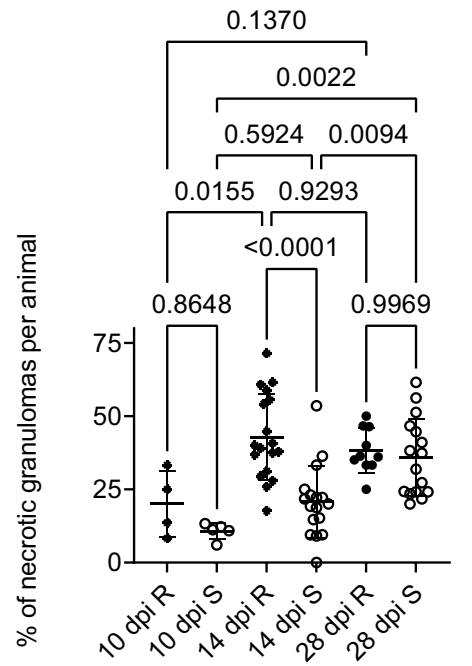

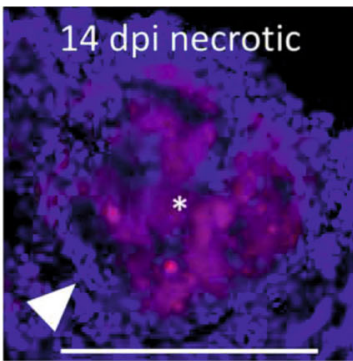
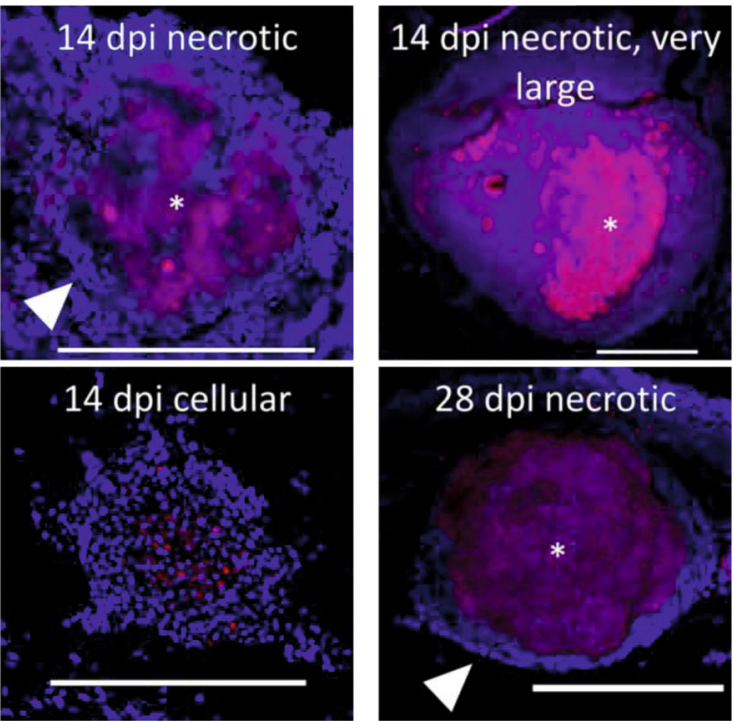

0.0332

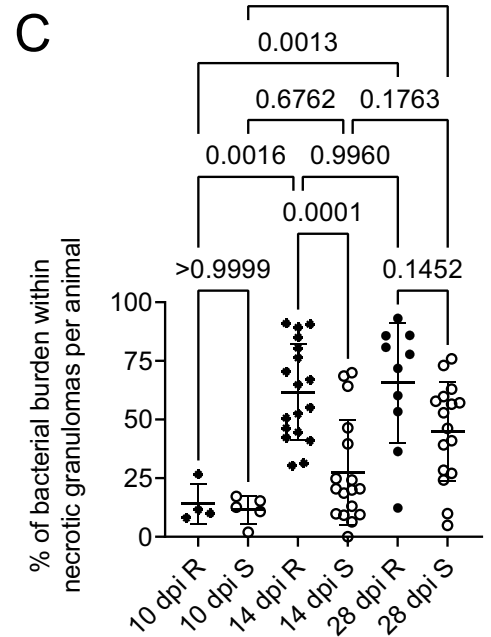

Fig. 2 Granuloma histopathology is accelerated during $\boldsymbol{M}$. abscessus $\mathbf{R}$ infection compared to S. A Stereotypical examples of bacterial lesions from DAPIstained sections from adult zebrafish infected with $M$. abscessus expressing tdTomato. Top row infected with the rough variant, bottom row infected with the smooth variant, timepoints as indicated. Images are representative of the two experimental replicates quantified in B, C. Scale bars indicate $200 \mu$ m. Filled arrowheads indicate epithelised macrophage nuclei forming a stereotypical concentric layer surrounding the mycobacterial core of necrotic

granulomas, * indicate necrotic cores. B Quantification of bacterial lesion necrosis in adult zebrafish infected with approximately $10^{5}$ CFU M. abscessus. Each data point represents the proportion of lesions from a single animal. C Quantification of bacterial burden stratified by lesion necrosis in adult zebrafish infected with either the R or the $\mathrm{S}$ variant of $M$. abscessus. Each data point represents the proportion of lesions from a single animal. Total individual lesions and animals analysed in B and C pooled from two experimental replicates (necrotic/unorganised/animals): $10 \mathrm{dpi}$ R (37/228/4); $10 \mathrm{dpi} \mathrm{S} \mathrm{(107/788/5);} 14$ dpi R (299/447/18); 14 dpi S (111/539/17); 28 dpi R (149/217/10); 28 dpi S (382/603/16). Statistical testing by two-sided ANOVA. Data are presented as mean values \pm SD. Source data are provided as a Source Data file.

and necrotic $M$. abscessus R granulomas (Fig. 5A). We did not observe T cell interaction with $M$. abscessus S growing "freely" around peritoneal organs early in infection (Fig. 5B) and the $\mathrm{T}$ cell response to tissue-invasive $M$. abscessus $\mathrm{S}$ was noticeably less than that for equivalent sized M. abscessus R granulomas (Fig. 5C).

To directly test the requirement of $\mathrm{T}$ cells for containing $M$. abscessus, we next utilised the T cell-deficient $l c k^{-/-s a 410}$ mutant line. We infected wild type (WT) control and $l c k^{-/-s a 410}$ mutant adult zebrafish with both the $\mathrm{S}$ and $\mathrm{R}$ variants. $\mathrm{T}$ cell-deficient adult zebrafish were significantly more susceptible to $M$. abscessus $\mathrm{R}$ infection with reduced survival over 28 days of infection $(P=0.0005$, Log-rank test) (Fig. 5D). T cell deficiency had a less pronounced effect on the survival of animals infected with $M$. abscessus $\mathrm{S}$ compared to $M$. abscessus $\mathrm{R}$ infection (WT $\mathrm{S}$ versus $\mathrm{lck}^{-1-} \mathrm{S}$; $P=0.03$, Log-rank test). Within the T cell-deficient animals, there was a 5.5 day increased median survival for $M$. abscessus S-infected animals (34 dpi) compared to M. abscessus R (28.5 dpi), although both groups eventually succumbed to infection at the same rate after 35 dpi ( $\mathrm{P}=0.78$, Log-rank test). Bacterial burden was significantly increased at $14 \mathrm{dpi}$ in $l c k^{-/-s a 410}$ animals infected with the $\mathrm{R}$, but not the $\mathrm{S}$ variant compared to burdens in WT adult zebrafish (Fig. 5E).

Surprisingly, we found necrotic granulomas in a survivor 56 dpi $l c k^{-/-s a 410}$ fish infected with $M$. abscessus R (Supplemental Fig. 3). These granulomas were all relatively small, with the largest having necrotic cores of approximately $100 \mu \mathrm{m}$, compared to the large granulomas seen in our $70 \mathrm{dpi}$ WT animals at more than $500 \mu \mathrm{m}$ (Supplemental Fig. 1).

These observations demonstrate that the control of $M$. abscessus $\mathrm{R}$ infection is more reliant on $\mathrm{T}$ cell-mediated immunity 

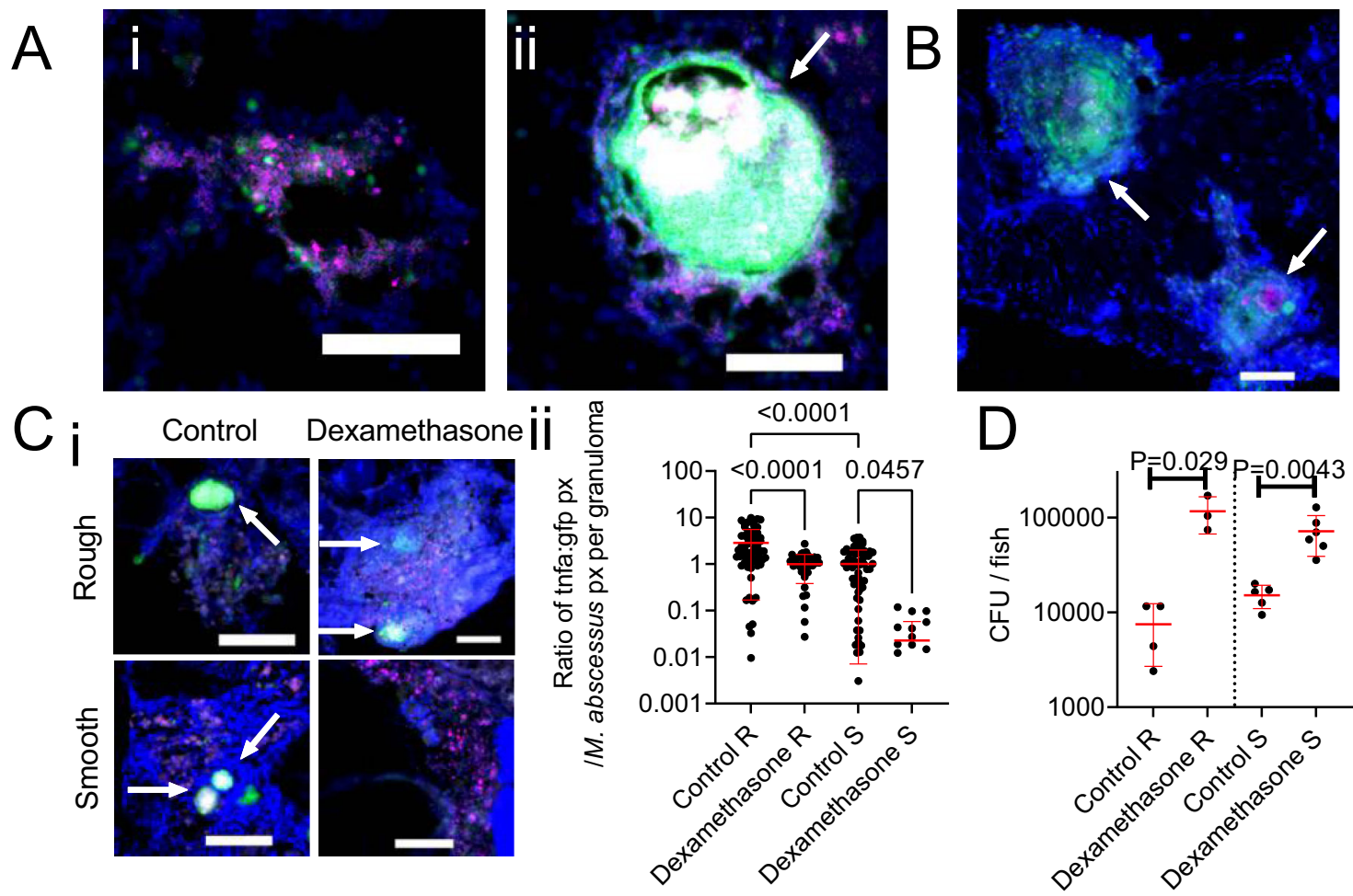

Fig. 3 Host tnfa expression is driven by $\boldsymbol{M}$. abscessus infection and control of $\boldsymbol{M}$. abscessus infection is sensitive to immunosuppression by dexamethasone. A Examples of $\mathrm{R}$ variant $M$. abscessus-tdTomato lesions in DAPI-stained cryosections from a 14 dpi TgBAC(tnfa:GFP)pd1028 adult zebrafish. i. Cellular lesion, ii. Necrotic granuloma. Arrow indicates necrotic granuloma, M. abscessus-tdTomato is coloured magenta, tnfa promoter induction is marked in green. Scale bars indicate $100 \mu \mathrm{m}$. Images are representative of two biological replicates of 3 animals per replicate. B Examples of $S$ variant $M$. abscessus-tdTomato lesions in DAPI-stained cryosections from a $28 \mathrm{dpi}$ TgBAC(tnfa:GFP)pd1028 adult zebrafish. Arrows indicate necrotic granulomas, M. abscessus-tdTomato is coloured magenta, tnfa promoter induction is marked in green. Scale bar indicates $100 \mu \mathrm{m}$. Images are representative of two biological replicates of 3 animals per replicate. C i. Examples of tnfa promoter activity in 14 dpi TgBAC(tnfa:GFP)pd1028 adult zebrafish infected with M. abscessus-tdTomato and treated with dexamethasone as indicated. Arrows indicate necrotic granulomas, M. abscessus-tdTomato is coloured magenta, tnfa promoter induction is marked in green. Scale bars indicate $100 \mu \mathrm{m}$. Control images are representative of 3 animals per M. abscessus variant, dexamethasone images are representative of 2 animals per $M$. abscessus variant. ii. Quantification of GFP pixels as a function of M. abscessus-tdTomato fluorescence in TgBAC(tnfa:GFP)pd1028 adult zebrafish. Data are a single experimental replicate, each point represents a single granuloma, total animals per group: Control $\mathrm{R}=3$, Dexamethasone $\mathrm{R}=2$, Control $\mathrm{S}=3$, Dexamethasone $\mathrm{S}=3$. Statistical testing by two-sided ANOVA. $\mathbf{D}$ Enumeration of $\mathrm{CFUs}$ from adult zebrafish infected with either the $\mathrm{R}$ or the $\mathrm{S}$ variant of $M$. abscessus and treated with dexamethasone. Each point represents a single animal and data are representative of 2 biological replicates. Total number of animals: Control $R=4$, Dexamethasone $R=3$, Control $S=5$, Dexamethasone $S=6$. Statistical testing by two-sided Student's $t$ test within each of the independent $R$ and $S$ experiments. Data are presented as mean values \pm SD. Source data are provided as a Source Data file.

than $M$. abscessus $\mathrm{S}$ infection during the initial 2 weeks of infection.

Regulatory T cells interact with rough $M$. abscessus granulomas to control infection burden. To further investigate the role of host immunopathology in $M$. abscessus infection, we next investigated the recruitment of regulatory $\mathrm{T}$ cells (Tregs) to sites of $M$. abscessus infection using the TgBAC(foxp3a:TagRFP,cryaa:EGFP) $)^{v c c 3}$ transgenic line where Tregs are labelled with RFP expression $^{33}$. Consistent with the recruitment profile of TgBAC(lck:EGFP) ${ }^{v c c 4}$ positive $\mathrm{T}$ cells, we observed Treg association and penetration throughout cellular and necrotic $M$. abscessus R granulomas from 14 dpi (Fig. 6A), and to necrotic $M$. abscessus $\mathrm{S}$ granulomas from $28 \mathrm{dpi}$ (Fig. 6B). The Treg response to $M$. abscessus $\mathrm{R}$ granulomas was significantly higher than for equivalent sized $M$. abscessus S granulomas at 14 dpi (Fig. 6C). Although we observed a higher Treg response to $M$. abscessus S than $\mathrm{R}$ at 28 dpi (Fig. 6C), this was driven by the significantly higher burden of $M$. abscessus $\mathrm{R}$ than $\mathrm{S}$ per granuloma rather than an absolute increase in Treg fluorescence per granuloma (28 dpi median M. abscessus fluorescence R: 2072, $n=66$; S: 41.00, $n=29$; Mann-Whitney $U$ test $P<0.0001$. Median Treg fluorescence R: $1298, n=66$; S: $123.0, n=29$; Mann-Whitney U test $P<0.0001)$.

As we had only observed significant Treg involvement with $M$. abscessus $\mathrm{R}$ granulomas, we next asked if hyperinflammatory foxp $3 a^{v c c 6}$ mutant zebrafish would have exacerbated M. abscessus R-infection. Homozygous mutant foxp $3 a^{v c c 6 / v c c 6}$ zebrafish had a higher infection burden than WT clutchmate controls (Fig. 6D). However, there were similar percentages of necrotic granulomas and similar burdens of $M$. abscessus within necrotic granulomas for each group at $14 \mathrm{dpi}$ (Fig. 6E, F).

\section{Discussion}

In this study, we report the use of adult zebrafish to probe both host and mycobacterial determinants of pathogenesis during persistent infection with M. abscessus. Infection with the R and S variants was maintained at high levels up to one month post infection in genetically intact animals, a major improvement on traditional mouse models of $M$. abscessus infection.

It is well known that the intracellular lifestyle of the $\mathrm{R}$ and $\mathrm{S}$ morphotypes differ significantly, resulting in entirely distinct 

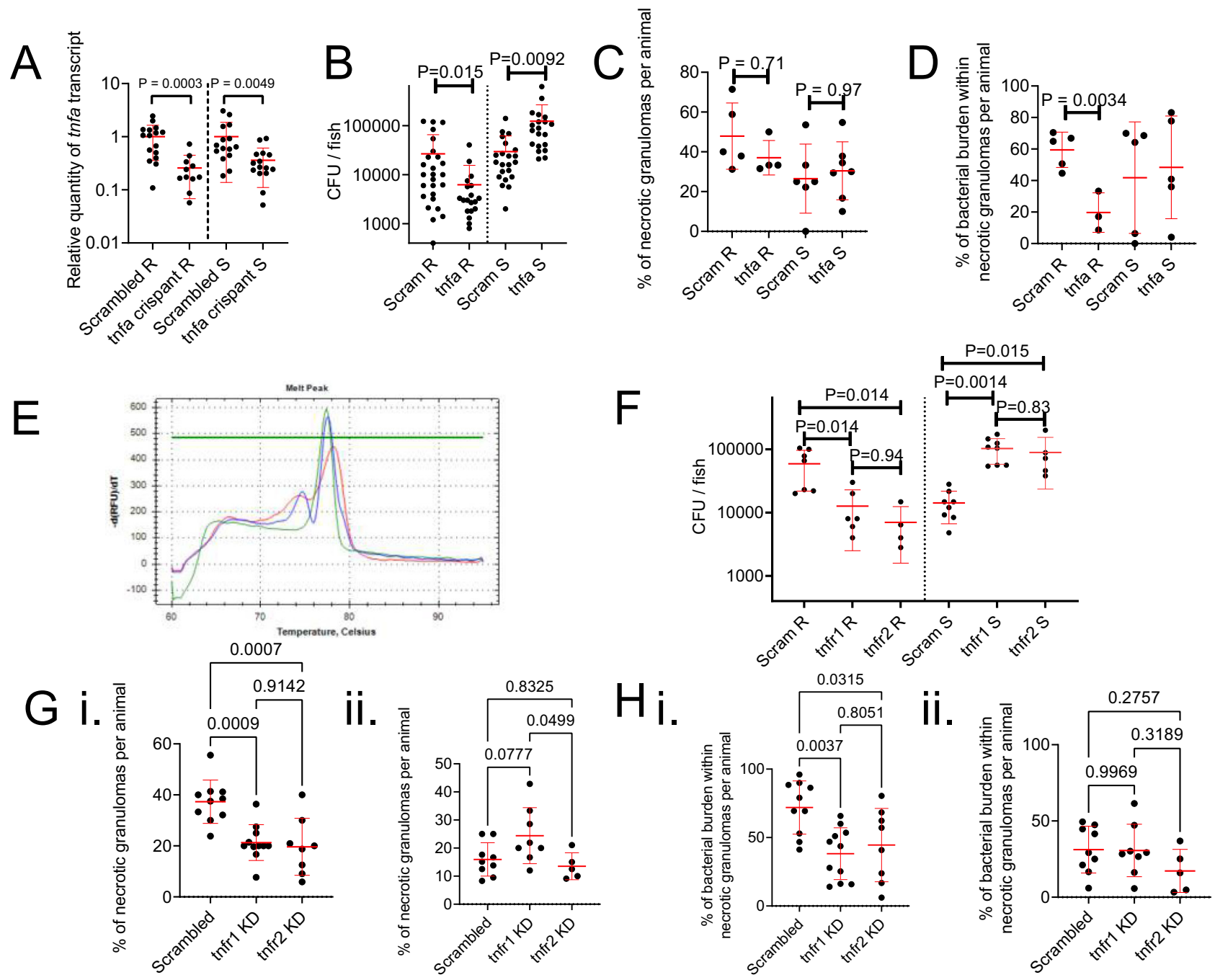

Fig. 4 Host TNF drives $\boldsymbol{M}$. abscessus R granuloma expansion and control of $\boldsymbol{M}$. abscessus $\mathbf{S}$ infection. A Quantification of tnfa transcripts activity in 14 dpi zebrafish injected with scrambled control or tnfa-targeting gRNA-Cas9 complexes. Each data point represents a single animal, data are pooled pooled from 2 biological replicates, total number of animals: Scrambled $R=16$, tnfa crispant $R=11$, Scrambled $S=15$, tnfa crispant $S=15$. Statistical testing by twosided Student's $t$ test within each of the independent R and S experiments. B Enumeration of CFUs from tnfa crispant adult zebrafish infected with $10^{4} \mathrm{CFU}$ of either the $\mathrm{R}$ or the $\mathrm{S}$ variant of $M$. abscessus. Each data point represents a single animal, data are pooled from 3 biological replicates, total number of animals: Scrambled $R=26$, tnfa crispant $R=19$, Scrambled $S=22$, tnfa crispant $S=20$. Statistical testing by two-sided Student's $t$ test within each of the independent $\mathrm{R}$ and $\mathrm{S}$ experiments. C Quantification of bacterial lesion necrosis in tnfa crispant adult zebrafish infected with $M$. abscessus. Each data point represents the proportion of lesions from a single animal. Statistical testing by two-sided ANOVA. D Quantification of bacterial burden stratified by lesion necrosis in tnfa crispant adult zebrafish infected with $M$. abscessus. Each data point represents the proportion of lesions from a single animal. Total individual lesions and animals analysed in C, D (necrotic/unorganised/animals): Scrambled R (43/51/5); tnfa crispant R (12/21/3); Scrambled S (25/43/ 5); tnfa crispant S (57/96/5). Statistical testing by two-sided ANOVA. E Example of high resolution melt analysis of the first tnfr2 CRISPR target site in 14 dpi zebrafish injected with scrambled control (blue), tnfr1 (green), or tnfr2 (red)-targeting gRNA-Cas9 complexes. F Enumeration of CFUs from tnfr1 and tnfr2 crispant adult zebrafish infected with $10^{4} \mathrm{CFU}$ of either the $\mathrm{R}$ or the $\mathrm{S}$ variant of $M$. abscessus. Each data point represents a single animal, data are representative of 3 biological replicates. Statistical testing by two-sided ANOVA within each of the independent $\mathrm{R}$ and $\mathrm{S}$ experiments. Total number of animals (Scram/tnfr1/tnfr2): $R=7 / 6 / 4, S=8 / 8 / 5$. G Quantification of bacterial lesion necrosis in tnfr1 and tnfr2 crispant adult zebrafish infected with $M$. abscessus. Each data point represents the proportion of lesions from a single animal. H. Quantification of bacterial burden stratified by lesion necrosis in tnfr 1 and tnfr2 crispant adult zebrafish infected with M. abscessus. Each data point represents the proportion of lesions from a single animal. Total individual lesions and animals analysed in G, H (necrotic/unorganised/animals): Scrambled R (91/224/10); tnfr1 crispant R (89/308/11); tnfr2 crispant R (40/173/ 8); Scrambled S (25/139/9); thfr1 crispant S (40/143/8); thfr2 crispant S (17/97/5). Statistical testing by two-sided ANOVA. Data are presented as mean values \pm SD. Source data are provided as a Source Data file.

infection scenarios that, we hypothesise, underlie the accelerated granuloma formation by the $\mathrm{R}$ variant in adult zebrafish ${ }^{10,34}$. The absence of GPL on the outer mycobacterial membrane causes corded growth of $\mathrm{R}$ variants, resulting in multiple bacilli being simultaneously phagocytosed by macrophages and overloaded phagosomes that rapidly activate autophagy pathways ${ }^{12,34}$. Comparatively, the $\mathrm{S}$ variant is able to survive for an extended period of time within the phagosome, producing a chronic and persistent infection ${ }^{10,15}$. As such, these polar infection responses may explain why the $\mathrm{R}$ variant displays widespread necrotic granuloma formation by $14 \mathrm{dpi}$, compared to the $\mathrm{S}$ variant that shows delayed onset of granuloma formation after $14 \mathrm{dpi}$. Moreover, this observation matches the superior in vivo growth performance of $\mathrm{S}$ bacilli compared to $\mathrm{R}$, suggesting that the $\mathrm{R}$ 

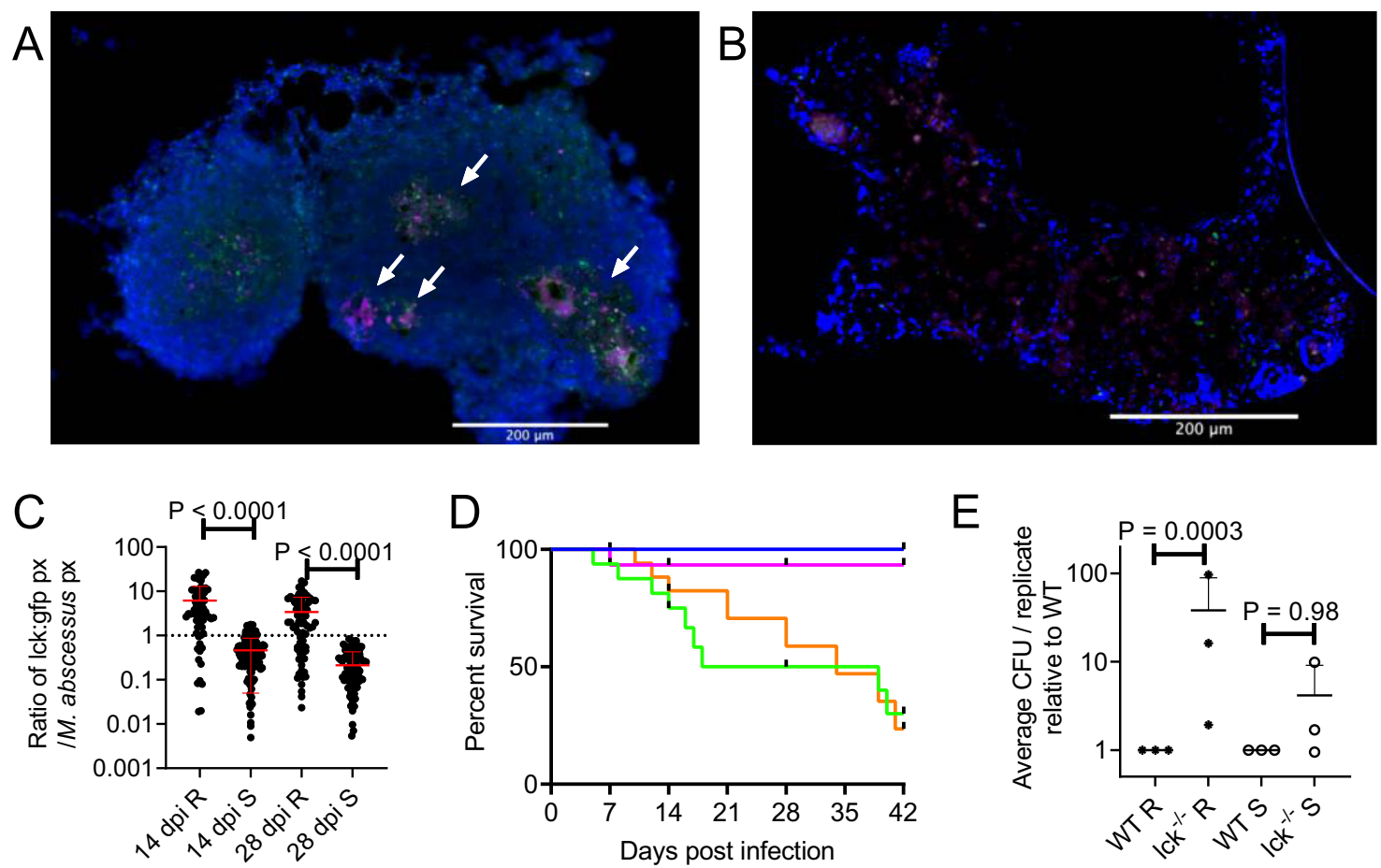

$$
\begin{array}{ll}
\perp \text { WT R } & \perp \text { WT S } \\
\perp \mathrm{Ick}^{-/-} \mathrm{R} & \perp \mathrm{Ick}^{-/-} \mathrm{S}
\end{array}
$$

Fig. 5 T cell-mediated immunity differentially controls infection by $\boldsymbol{M}$. abscessus variants. A Examples of $T$ cell recruitment to granulomas in 14 dpi TgBAC(Ick:EGFP) vcc4 adult zebrafish infected with R M. abscessus-tdTomato. B Example of lack of T cell recruitment to S M. abscessus- tdTomato in a large cellular lesion from a 14 dpi TgBAC(Ick:EGFP)vcc4 adult zebrafish. Scale bars indicate $200 \mu \mathrm{m}$. Arrows indicate necrotic granulomas, M. abscessus-tdTomato is coloured magenta, Ick:gfp positive T cells are marked in green. Images from $\mathbf{A}$ and $\mathbf{B}$ are representative of the dataset analysed in $\mathbf{C}$. C Quantification of T cell GFP pixels as a function of M. abscessus-tdTomato fluorescence in TgBAC(Ick:EGFP)vcc4 adult zebrafish. Each point represents a single lesion. Total $n$ per group (lesions/animals): 14 dpi $R=74 / 2, S=137 / 3 ; 28$ dpi $R=91 / 3, S=117 / 2$. D Survival analysis of WT and Ick ${ }^{-/}-$sa410 adult zebrafish infected with R or S M. abscessus. Total $n=12$ WT/Mabs R; $16 \mathrm{lck}^{-/-} /$Mabs R; 15 WT/Mabs S; 22 lck $\mathrm{ll}^{-/}$/Mabs S. E Normalised CFUs recovered from 14 dpi WT and $\mathrm{lck}^{-/-}$sa410 adult zebrafish infected with M. abscessus. Each point represents the average of a single experiment with at least 2 animals per group. Total $n$ per group: $\mathrm{R} W T=12 \mathrm{lck}^{-/-}=15 ; \mathrm{S} W T=11 \mathrm{lck}^{-/-}=12$. Statistical testing by two-sided ANOVA. Data are presented as mean values \pm SD. Source data are provided as a Source Data file.

variant is at an overall disadvantage because of its intrinsic hyperinflammatory status and the activation of $\mathrm{T}$ cell-mediated immunity that occurs concomitantly with granuloma formation. Interestingly, earlier reports using the zebrafish embryo demonstrated that both bacterial burden and granuloma formation dynamics were similar between both the $S$ and $R$ variants ${ }^{10,26}$, highlighting the critical role of adaptive immunity in divergent responses to the M. abscessus variants. Taken together, our data provide additional evidence for the distinct intracellular fates of both $S$ and $R$ variants in vivo, and further implicate the role of adaptive immunity in granuloma formation and the control of $M$. abscessus infection in adult zebrafish.

While dexamethasone-sensitive immunity was necessary for the control of both variants and Treg immunomodulation was necessary for the control of $\mathrm{R}$ infection, we discovered a striking pathogenic role for $t n f a$, $t n f r 1$, and $t n f r 2$ during $\mathrm{R}$ infections and a protective role during $\mathrm{S}$ infections. Although dexamethasone treatment marginally reduced host tnfa promoter activity around sites of infection, dexamethasone treatment has wider immunosuppressive effects. Our data points towards a specific role for host TNF signalling being co-opted by $M$. abscessus $\mathrm{R}$ infection to drive to the formation of necrotic granulomas as a permissive niche for bacterial growth within the host. The detrimental role of tnfa, tnfrl, and $t$ nfr 2 in adult zebrafish-M. abscessus $\mathrm{R}$ infection is the opposite of previously published work in the zebrafish embryo using knockdown of $\operatorname{tnfr} 1^{23}$, which points to the importance of using multiple models systems to investigate different stages of infection. Our data suggest human studies of increased susceptibility to M. abscessus in patients receiving TNF inhibition should be stratified by strain morphology to investigate the clinical relevance of this effect. We hypothesise that the increased susceptibility of patients receiving TNF inhibition therapy to $M$. abscessus disease will be driven by the loss of control of $\mathrm{S}$ variant infections ${ }^{35}$.

Our finding that Tregs are closely associated with M. abscessus $\mathrm{R}$ granulomas and act to restrain $M$. abscessus $\mathrm{R}$ growth provides complementary evidence that $M$. abscessus $\mathrm{R}$ takes advantage of host immunopathogenesis to establish chronic infection in adult zebrafish. While the assays proved adept at detecting reduced necrosis in the tnfa, tnfrl, and tnfr2 crispants, Treg-deficient mutants had comparable proportions of necrotic granulomas and proportions of M. abscessus R within necrotic granulomas to WT clutchmates indicating no increase in necrotic granuloma phenotypes. We hypothesise that this is because our granuloma necrosis assays for $M$. abscessus $\mathrm{R}$ are running at the upper limits of signal. Zebrafish Treg cells produce context-specific regulatory factors in a Foxp3a-dependent manner ${ }^{36}$, and future experiments are warranted to identify the protective Foxp3a-dependent factors produced in the context of $M$. abscessus $\mathrm{R}$ granulomas.

$\mathrm{T}$ cells are critical host determinants in the control of mycobacterial infection ${ }^{37}$. Recruitment of $\mathrm{T}$ cells into granulomas is thought to be essential in containing persistent infection, while $\mathrm{T}$ 

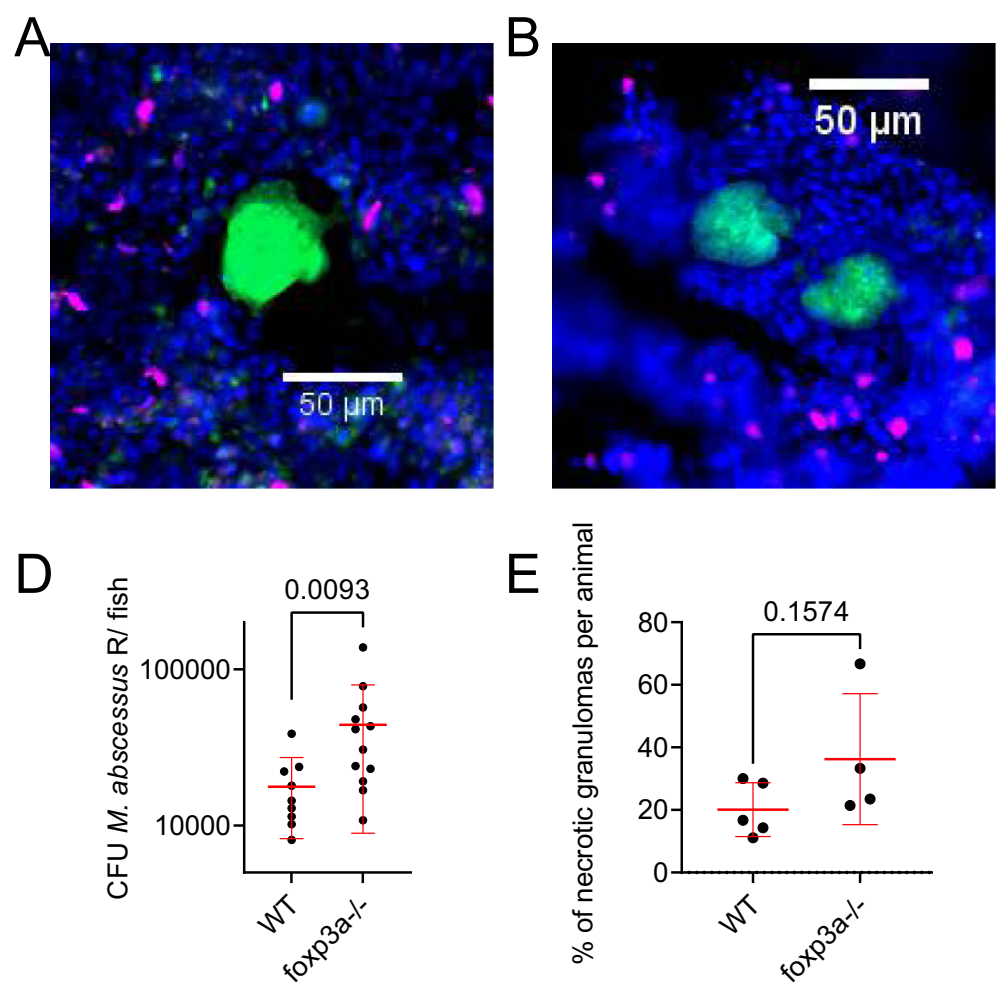
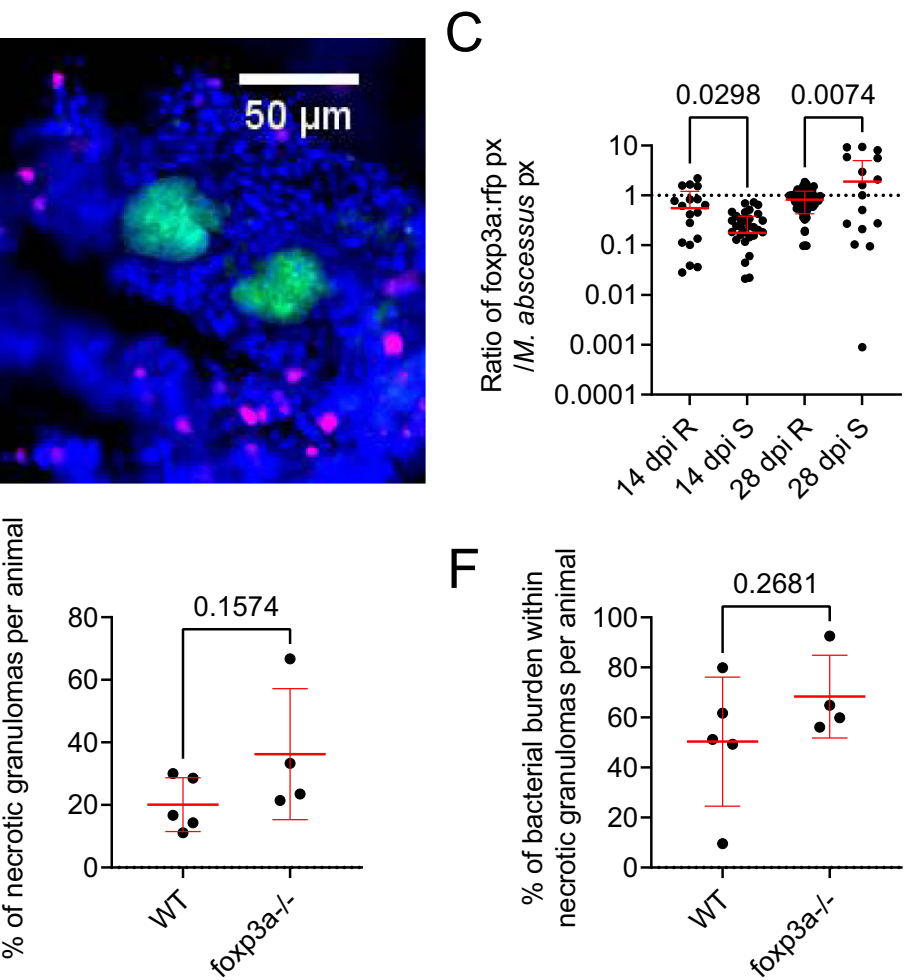

Fig. 6 Regulatory $\mathbf{T}$ cells interact with rough $\boldsymbol{M}$. abscessus granulomas to control infection burden. A Example of Treg recruitment to a necrotic $R$ M. abscessus- wasabi granuloma in 14 dpi TgBAC(foxp3a:TagRFP,cryaa:EGFP)vcc3 adult zebrafish. B Example of Treg recruitment to a necrotic S M. abscessuswasabi granuloma from a $28 \mathrm{dpi}$ TgBAC(foxp3a:TagRFP, cryaa:EGFP)vcc3 adult zebrafish. Scale bars indicate $50 \mu \mathrm{m}$. M. abscessus-wasabi is coloured green, foxp3a:TagRFP positive Treg are marked in magenta. Images from A and B are representative of the dataset analysed in C. C Quantification of Treg RFP pixels as a function of $M$. abscessus-wasabi fluorescence in TgBAC(foxp3a:TagRFP,cryaa:EGFP)vcc3 adult zebrafish. Each point represents a single lesion. Total n per group (lesions/animals): 14 dpi $R=26 / 4, S=55 / 3 ; 28$ dpi $R=66 / 3, S=29 / 3$. Statistical testing by 2-way ANOVA. D Enumeration of CFUs from foxp $3 a^{v c c 6}$ mutant adult zebrafish infected with $2 \times 10^{4}$ CFU M. abscessus R. Each data point represents a single animal, data are representative of two biological replicates. Statistical testing by two-sided $T$ test. Total number of animals: WT $=9$, foxp3a- $/-=12$. E Quantification of bacterial lesion necrosis in foxp $3 a^{v c c 6}$ mutant adult zebrafish infected with M. abscessus R. Each data point represents the proportion of lesions from a single animal. F Quantification of bacterial burden stratified by lesion necrosis in foxp3avcc6 mutant adult zebrafish infected with M. abscessus R. Each data point represents the proportion of lesions from a single animal. Total individual lesions and animals analysed in E, F (necrotic/unorganised/animals): WT (35/ 91/5); foxp3avec6 mutant (22/97/4). Statistical testing by two-sided T test. Data are presented as mean values \pm SD. Source data are provided as a Source Data file.

cell deficiencies are associated with greater mycobacterial infection severities ${ }^{27,37-39}$. Recently, an adult zebrafish infection model for M. leprae demonstrated that $\mathrm{T}$ cells are essential for containment of infection ${ }^{27}$. Herein, we examined the recruitment of $\mathrm{T}$ cells within granulomas and identified that $\mathrm{S}$ variant granuloma were characterised by a relative paucity of $\mathrm{T}$ cell infiltration, suggesting that $\mathrm{T}$ cells play a less significant role in $\mathrm{S}$ variant infections. Consistent with this, we observed increased in vivo growth of the $\mathrm{R}$, but not $\mathrm{S}$, variant in the absence of $\mathrm{T}$ cells when compared to WT animals and in our co-infection experiments where $\mathrm{R}$ but not $\mathrm{S}$ burdens were increased in $l c k^{-1-}$ zebrafish. Following the hypothesis that patients acquire an environmental $S$ variant of $M$. abscessus, our findings provide insight into the clinical observation that AIDS patients are not at increased risk of M. abscessus infection to the same degree that AIDS is a risk factor for M. tuberculosis and other non-tuberculous mycobacterium infections, such as Mycobacterium avium, as $\mathrm{T}$ cell deficiency has a limited effect on the control of $S$ variant infection ${ }^{37}$.

The extended maintenance of $\mathrm{R}$ variant burden for at least 4 weeks in zebrafish is comparable to our recent data from the $\mathrm{C} 3 \mathrm{HeB} / \mathrm{FeJ}$ mouse ${ }^{40}$, but the proliferation of the $\mathrm{S}$ variant up to a $\log$ above inoculation dose is unprecedented in a genetically intact vertebrate host. The granulomatous immunopathology in mycobacterium-infected $\mathrm{C} 3 \mathrm{HeB} / \mathrm{FeJ}$ mice is due to an exaggerated type I interferon response suppressing protective IL-1 ${ }^{41}$. Further analysis of IFN and IL-1 responses to M. abscessus infection of mice and zebrafish will help translate our understanding of these dichotomous responses into host directed therapies.

We did not observe switching of $S M$. abscessus into a rough colony morphotype at any timepoint during this study. In vivo switching is a rare event that has only been documented in immunocompromised mice or after months-to-years of infection in patients ${ }^{13,42}$. The high $S$ morphotype burdens achieved in adult zebrafish suggest this platform may be useful for future studies of switching during extended infections, with the potential to model responses to chemotherapy and transcriptional adaptations to host residence that may include rough-like surface morphologies ${ }^{13,43}$.

To date, our understanding of the diverse immune responses between $\mathrm{S}$ and $\mathrm{R}$ variants have essentially been thoroughly described with respect to innate immunity, and currently our knowledge pertaining to adaptive immunity in M. abscessus infection has been poorly characterised ${ }^{20}$. Using this new adult zebrafish $M$. abscessus infection model, we have shown that $S$ and 
$\mathrm{R}$ variants produce strikingly different disease phenotypes, driven by unique interactions with host immunity. Consequently, these results suggest that the host-pathogen interactions dictating $M$. abscessus pathogenesis are complex and implicate different arms of host immunity to a greater extent than originally anticipated. Future work should exploit this relevant animal model in combination with zebrafish lacking the CFTR gene, and for the development and testing of novel antibiotics and vaccine candidates that may be used for the treatment of M. abscessus infection. The robust infection phenotypes created by exemplar R and $\mathrm{S} M$. abscessus strains suggest adult zebrafish will be an important platform to study M. abscessus virulence factors and clinical strain genetic variation.

\section{Methods}

Zebrafish strains and handling. Zebrafish strains used in this study were $A B$ strain wildtype, TgBAC(tnfa:GFP)pd1028, TgBAC(lck:EGFP) ${ }^{v c c 4}, l c k^{-/-s a 410}$ mutant, TgBAC(foxp3a:TagRFP,cryaa:EGFP) $)^{v c c 3}$, foxp $3 a^{v c c 6}$ mutant ${ }^{31,33}$ between 3 and 6 months of age. Animals were held in a $28^{\circ} \mathrm{C}$ incubator with a $14: 10 \mathrm{~h}$ light:dark cycle. Animals were infected by intraperitoneal injection with approximately $10^{5}$ CFU M. abscessus, unless otherwise stated, using a $31 \mathrm{G}$ insulin needle and syringe, as previously described ${ }^{44}$. Initial inoculum dose was confirmed by plating of inoculum dose and homogenisation of injected fish immediately after infection. Infected zebrafish were recovered into system water (approximately $750 \mu$ s conductivity, $\mathrm{pH}$ 7.4) and held in $1 \mathrm{~L}$ beakers at a density of approximately one fish per $50-100 \mathrm{ml}$ water. Animals were cleaned daily with a $20 \%$ water change and fed once per day for the duration of experiments as previously described ${ }^{44}$. Infection experiments were carried out with ethical approval from the Sydney Local Health District Animal Welfare Committee approval 16-037.

The vcc allele zebrafish lines are available on request from the corresponding author (Sydney, Australia or Singapore) or the creator Dr Kazu Kikuchi (current address Osaka, Japan). The $\mathrm{lck}^{--}{ }^{-s a 410}$ zebrafish line is a publicly available resource from the Zebrafish International Resource Center (Oregon, USA). The $\operatorname{TgBAC}(\operatorname{tnfa}: G F P)^{p d 1028}$ zebrafish line is available from the corresponding author or the creators at Duke University School of Medicine (North Carolina, USA).

M. abscessus strains and handling. Rough (R) and smooth (S) variants of $M$. abscessus strain CIP $104536^{\mathrm{T}}$ were grown at $37^{\circ} \mathrm{C}$ in Middlebrook $7 \mathrm{H} 9$ broth supplemented with $10 \%$ Oleic acid/Albumin/Dextrose/Catalase (OADC) enrichment and $0.05 \%$ Tween 80 or on Middlebrook 7 H10 agar containing $10 \%$ OADC (7H10 OADC). Recombinant M. abscessus strains expressing tdTomato or Wasabi were grown in the presence of $500 \mu \mathrm{g} / \mathrm{ml}$ hygromycin ${ }^{10,23}$. Homogenous bacterial suspensions for intraperitoneal injection in adult fish were prepared as previously reported ${ }^{45}$. Briefly, M. abscessus was plated from freezer stocks onto 7H10 OADC supplemented with $500 \mu \mathrm{g} / \mathrm{ml}$ hygromycin. Colonies were picked and outgrown in 7H9 OADC $500 \mu \mathrm{g} / \mathrm{ml}$ hygromycin for three days. M. abscessus was harvested by pelleting, resuspended in $7 \mathrm{H} 9$ and sheered through at $27 \mathrm{G}$ needle prior to aliquoting and freezing.

The fluorescent $M$. abscessus strains are available from the corresponding author or their creator Dr Laurent Kremer at IRIM (Montpellier, France).

Bacterial recovery. Animals were killed by tricaine anaesthetic overdose $(>300 \mu \mathrm{g} /$ $\mathrm{ml}$ ) and rinsed in sterile water. Individual carcasses were mechanically homogenised and serially diluted into sterile water. Homogenates were plated onto $7 \mathrm{H} 10$ supplemented with OADC and $300 \mu \mathrm{g} / \mathrm{ml}$ hygromycin. Plates were grown for at least 4 days at $37^{\circ} \mathrm{C}$.

Histology. Animals were subjected to cryosectioning as previously described ${ }^{44}$. Briefly, euthanasia was performed by tricaine anaesthetic overdose and specimens were fixed for 2-4 days in $10 \%$ neutral buffered formalin at $4{ }^{\circ} \mathrm{C}$. Specimens were then rinsed in PBS, incubated overnight in 30\% sucrose, incubated overnight in 50/ 50 30\% sucrose and Tissue-Tek O.C.T. compound (OCT, Sakura Finetek), and finally incubated overnight in OCT prior to freezing at $-80^{\circ} \mathrm{C}$. Cryosectioning was performed to produce $20 \mu \mathrm{m}$ thick sections. Sections were post-fixed in $10 \%$ neutral buffered formalin and rinsed in PBS prior to further processing. Slides for fluorescent imaging were mounted with coverslips using Fluoromount G containing DAPI (Proscitech).

$\mathrm{T}$ cells were detected in sections from $\operatorname{TgBAC}(\text { lck:EGFP) })^{v c c 4}$ zebrafish by antiGFP staining to enhance visible fluorescent green signal (primary antibody: ab13970, Abcam; secondary antibody: ab150173, Abcam), both antibodies were diluted 1:500 in 5\% goat serum (Thermofisher). Stained slides were then mounted with coverslips using Fluoromount G containing DAPI. All imaging was carried out on a Leica DM6000B microscope with LAS software version X (Leica) and image analysis was performed in ImageJ version $1.53 \mathrm{n}(\mathrm{NIH})$.
Drug treatment. Animals for dexamethasone treatment were infected with $1-5 \times 10^{4} \mathrm{CFU}$ to ensure survival following immunosuppression. Water soluble dexamethasone (Sigma, D2915) was added to a final concentration of $40 \mu \mathrm{g} / \mathrm{ml}$ immediately following infection and changed every second day.

CRISPR-Cas9 gene knockdown. Embryos were injected at the single cell stage with injection mix containing $1 \mu \mathrm{l}$ phenol red, $2 \mu \mathrm{l} 500 \mathrm{ng} / \mu \mathrm{l}$ pooled guides, and $2 \mu \mathrm{l}$ $10 \mu \mathrm{M}$ Cas9. Control embryos are injected with scrambled guide RNA as previously described $^{46}$. The oligo sequences used to generate tnfa-targeting guides were computed by $\mathrm{Wu}$ et al. ${ }^{46}$ : guide 1 TAATACGACTCACTATAGGTTGAGAGTCG GGCGTTTTGTTTTAGAGCTAGAAATAGC, guide 2 TAATACGACTCACTAT AGGTCTGCTTCACGCTCCATAGTTTTAGAGCTAGAAATAGC, guide 3 TAA TACGACTCACTATAGGGATTATCATTCCCGATGAGTTTTAGAGCTAGAA ATAGC, guide 4 TAATACGACTCACTATAGGTCCTGCGTGCAGATTGAGGT TTTAGAGCTAGAAATAGC. Oligos for $t n f r 1$-targeting guides: guide 1 TAATA CGACTCACTATAGGGTGATGAAGGAACCTACCGTTTTAGAGCTAGAAAT AGC, guide 2 TAATACGACTCACTATAGGGTGAAGACTCCCTGGAATGTTT TAGAGCTAGAAATAGC, guide 3 TAATACGACTCACTATAGGCATTTTTA TGGCATACGAGTTTTAGAGCTAGAAATAGC, guide 4 TAATACGACTCACT ATAGGTCTTTAAATCGTCCGATAGTTTTAGAGCTAGAAATAGC. Oligos for tnfr2-targeting guides guide 1 TAATACGACTCACTATAGGTCTGAGTACATTC CATCTGTTTTAGAGCTAGAAATAGC, guide 2 TAATACGACTCACTATAG GTTCTTTGAGCGCCTTCGCGTTTTAGAGCTAGAAATAGC, guide 3 TAATA CGACTCACTATAGGTGCCTCTTACTCGGTGTGGTTTTAGAGCTAGAAATA GC, guide 4 TAATACGACTCACTATAGGGTCATGTAGTCAGGCGGAGTTTT AGAGCTAGAAATAGC.:

Embryos were raised to 10 weeks post fertilisation and were then infected with a reduced burden of $1-5 \times 10^{4} \mathrm{CFU}$, scaled to their smaller size.

Genotyping of $t n f r 1$ and $t n f r 2$ crispants was performed by high resolution melt analysis of amplicons produced by amplification of gDNA from diluted homogenate using oligos: $t n f r 1$ gFw GCAGTGCAGAAAACATGAGG, GRv CGTTTTGTGCATTGCTGGC; $t n f r 2$ gFw ACTCGCTTGTCTGTGCAATG, gRv TGGACACTTGAAACAATTGGGA. Amplification and analysis was carried out with MeltDoctor ${ }^{\text {TM }}$ HRM Master Mix (Thermofisher) reagent on a CFX thermocycler with CFX Maestro software version 2.2 (Biorad) according to MeltDoctor product specifications.

Phenotyping of $\operatorname{tnfa}$ crispants was performed by RT-qPCR using oligos: $\operatorname{tnfa}$ qFw GCTTATGAGCCATGCAGTGA, qRv AAGTGCTGTGGTCGTGTCTG; $18 \mathrm{~s}$ qFw TCGCTAGTTGGCATCGTTTATG, qRv CGGAGGTTCGAAGACGATCA. Homogenate was processed for RNA extraction following product specification for Trizol LS (Thermofisher). Reverse transcription was carried out from $2 \mu \mathrm{g}$ total RNA with the Applied Biosystems ${ }^{\mathrm{Tm}}$ High Capacity cDNA Reverse Transcription Kit (Thermofisher). Quantitative PCR was carried out with PowerUp ${ }^{\text {TM }}$ SYBR $^{\text {rM }}$ Green Master Mix (Thermofisher) on a CFX thermocycler with CFX Maestro software version 2.2 (Biorad).

Statistics. All statistical testing was carried out using Graphpad Prism version 9.3.1. Statistical tests are indicated in the figure legends and include both $\mathrm{T}$ tests for pairwise comparisons and ANOVA analyses with post hoc analysis for comparison of three or more groups. Each data point indicates a single animal unless otherwise stated.

Reporting summary. Further information on research design is available in the Nature Research Reporting Summary linked to this article.

\section{Data availability}

The processed analysis data are included in the Source Data file that is provided with this paper. Raw image files will be archived for 10 years (2022-2032) by The Centenary Institute (Sydney, Australia). The raw image and analysis data are available under restricted access due to their large size and prohibitive cost of public hosting: they can be obtained upon request by email from the corresponding author, who will mediate access from the Centenary Institute servers. Expected response times will be within one week. Source data are provided with this paper.

Received: 18 August 2020; Accepted: 3 February 2022; Published online: 17 February 2022

\section{References}

1. Johansen, M. D., Herrmann, J. L. \& Kremer, L. Non-tuberculous mycobacteria and the rise of Mycobacterium abscessus. Nat. Rev. Microbiol., https://doi.org/ 10.1038/s41579-020-0331-1 (2020)

2. Medjahed, H., Gaillard, J. L. \& Reyrat, J. M. Mycobacterium abscessus: a new player in the mycobacterial field. Trends Microbiol. 18, 117-123 (2010). 
3. Tomashefski, J. F. Jr., Stern, R. C., Demko, C. A. \& Doershuk, C. F. Nontuberculous mycobacteria in cystic fibrosis. An autopsy study. Am. J. Respir. Crit. Care Med. 154, 523-528 (1996).

4. Nessar, R., Cambau, E., Reyrat, J. M., Murray, A. \& Gicquel, B. Mycobacterium abscessus: a new antibiotic nightmare. J. Antimicrobial Chemother. 67, 810-818 (2012).

5. Ferro, B. E. et al. Failure of the Amikacin, cefoxitin, and clarithromycin combination regimen for treating pulmonary Mycobacterium abscessus infection. Antimicrob. Agents Chemother. 60, 6374-6376 (2016).

6. Howard, S. T. et al. Spontaneous reversion of Mycobacterium abscessus from a smooth to a rough morphotype is associated with reduced expression of glycopeptidolipid and reacquisition of an invasive phenotype. Microbiology 152, 1581-1590 (2006).

7. Catherinot, E. et al. Acute respiratory failure involving an $\mathrm{R}$ variant of Mycobacterium abscessus. J. Clin. Microbiol. 47, 271-274 (2009).

8. Esther, C. R. Jr., Esserman, D. A., Gilligan, P., Kerr, A. \& Noone, P. G. Chronic Mycobacterium abscessus infection and lung function decline in cystic fibrosis. J. Cyst. Fibros. 9, 117-123 (2010).

9. Jonsson, B. E. et al. Molecular epidemiology of Mycobacterium abscessus, with focus on cystic fibrosis. J. Clin. Microbiol. 45, 1497-1504 (2007).

10. Bernut, A. et al. Mycobacterium abscessus cording prevents phagocytosis and promotes abscess formation. Proc. Natl Acad. Sci. USA 111, E943-E952 (2014).

11. Medjahed, H. \& Reyrat, J. M. Construction of Mycobacterium abscessus defined glycopeptidolipid mutants: comparison of genetic tools. Appl. Environ. Microbiol. 75, 1331-1338 (2009).

12. Gutierrez, A. V., Viljoen, A., Ghigo, E., Herrmann, J. L. \& Kremer, L. Glycopeptidolipids, a double-edged sword of the Mycobacterium abscessus complex. Front Microbiol. 9, 1145 (2018).

13. Pawlik, A. et al. Identification and characterization of the genetic changes responsible for the characteristic smooth-to-rough morphotype alterations of clinically persistent Mycobacterium abscessus. Mol. Microbiol. 90, 612-629 (2013).

14. Le Moigne, V. et al. Lsr2 is an important determinant of intracellular growth and virulence in Mycobacterium abscessus. Front Microbiol. 10, 905 (2019).

15. Bernut, A., Herrmann, J. L., Ordway, D. \& Kremer, L. The diverse cellular and animal models to decipher the physiopathological traits of Mycobacterium abscessus Infection. Front Cell Infect. Microbiol 7, 100 (2017).

16. Bernut, A. et al. In vivo assessment of drug efficacy against Mycobacterium abscessus using the embryonic zebrafish test system. Antimicrob. Agents Chemother. 58, 4054-4063 (2014).

17. Obregon-Henao, A. et al. Susceptibility of Mycobacterium abscessus to antimycobacterial drugs in preclinical models. Antimicrob. Agents Chemother. 59, 6904-6912 (2015).

18. Maggioncalda, E. C. et al. A mouse model of pulmonary Mycobacteroides abscessus infection. Sci. Rep. 10, 3690 (2020).

19. Riva, C. et al. A New Model of Chronic Mycobacterium abscessus Lung Infection in Immunocompetent Mice. Int J Mol Sci 21, https://doi.org/ $10.3390 /$ ijms 21186590 (2020).

20. Rottman, M. et al. Importance of T cells, gamma interferon, and tumor necrosis factor in immune control of the rapid grower Mycobacterium abscessus in C57BL/6 mice. Infect. Immun. 75, 5898-5907 (2007).

21. Oh, C. T., Moon, C., Jeong, M. S., Kwon, S. H. \& Jang, J. Drosophila melanogaster model for Mycobacterium abscessus infection. Microbes Infect. 15, 788-795 (2013).

22. Meir, M., Grosfeld, T. \& Barkan, D. Establishment and validation of galleria mellonella as a novel model organism to study Mycobacterium abscessus infection, pathogenesis, and treatment. Antimicrob Agents Chemother. 62, https://doi.org/10.1128/AAC.02539-17 (2018).

23. Bernut, A. et al. Mycobacterium abscessus-induced granuloma formation is strictly dependent on TNF signaling and neutrophil trafficking. PLoS Pathog. 12, e1005986 (2016).

24. Dubois, V. et al. MmpL8MAB controls Mycobacterium abscessus virulence and production of a previously unknown glycolipid family. Proc. Natl Acad. Sci. USA 115, E10147-E10156 (2018).

25. Halloum, I. et al. Deletion of a dehydratase important for intracellular growth and cording renders rough Mycobacterium abscessus avirulent. Proc. Natl Acad. Sci. USA 113, E4228-E4237 (2016).

26. Bernut, A. et al. CFTR protects against Mycobacterium abscessus infection by fine-tuning host oxidative defenses. Cell Rep. 26, 1828-1840 (2019). e1824.

27. Madigan, C. A., Cameron, J. \& Ramakrishnan, L. A Zebrafish model of Mycobacterium leprae granulomatous infection. J. Infect. Dis. 216, 776-779 (2017).

28. Oehlers, S. H. et al. Interception of host angiogenic signalling limits mycobacterial growth. Nature 517, 612-615 (2015)

29. Swaim, L. E. et al. Mycobacterium marinum infection of adult zebrafish causes caseating granulomatous tuberculosis and is moderated by adaptive immunity. Infect. Immun. 74, 6108-6117 (2006).
30. Parikka, M. et al. Mycobacterium marinum causes a latent infection that can be reactivated by gamma irradiation in adult Zebrafish. PLoS Pathog. 8 , e1002944 (2012).

31. Marjoram, L. et al. Epigenetic control of intestinal barrier function and inflammation in zebrafish. Proc. Natl Acad. Sci. USA 112, 2770-2775 (2015).

32. Myllymaki, H., Niskanen, M., Luukinen, H., Parikka, M. \& Ramet, M. Identification of protective postexposure mycobacterial vaccine antigens using an immunosuppression-based reactivation model in the zebrafish. Dis. Model. Mech. 11, https://doi.org/10.1242/dmm.033175 (2018).

33. Sugimoto, K., Hui, S. P., Sheng, D. Z., Nakayama, M. \& Kikuchi, K. Zebrafish FOXP3 is required for the maintenance of immune tolerance. Dev. Comp. Immunol. 73, 156-162 (2017).

34. Roux, A.-L. et al. The distinct fate of smooth and rough Mycobacterium abscessus variants inside macrophages. Open biology 6, https://doi.org/ 10.1098/rsob.160185 (2016).

35. Chan, E. D., Bai, X., Kartalija, M., Orme, I. M. \& Ordway, D. J. Host immune response to rapidly growing mycobacteria, an emerging cause of chronic lung disease. Am. J. Respir. Cell Mol. Biol. 43, 387-393 (2010).

36. Hui, S. P. et al. Zebrafish regulatory T cells mediate organ-specific regenerative programs. Dev. Cell 43, 659-672 (2017). e655.

37. Collins, F. M. Mycobacterial disease, immunosuppression, and acquired immunodeficiency syndrome. Clin. Microbiol Rev. 2, 360-377 (1989).

38. Mogues, T., Goodrich, M. E., Ryan, L., LaCourse, R. \& North, R. J. The relative importance of $\mathrm{T}$ cell subsets in immunity and immunopathology of airborne Mycobacterium tuberculosis infection in mice. J. Exp. Med. 193, 271-280 (2001).

39. Yang, J. D. et al. Mycobacterium tuberculosis-specific CD4+ and CD8+ T cells differ in their capacity to recognize infected macrophages. PLoS Pathog. 14, e1007060 (2018).

40. Le Moigne, V. et al. Efficacy of bedaquiline, alone or in combination with imipenem, against Mycobacterium abscessus in $\mathrm{C} 3 \mathrm{HeB} / \mathrm{FeJ}$ Mice. Antimicrob Agents Chemother. 64, https://doi.org/10.1128/AAC.00114-20 (2020).

41. Ji, D. X. et al. Type I interferon-driven susceptibility to Mycobacterium tuberculosis is mediated by IL-1Ra. Nat. Microbiol 4, 2128-2135 (2019).

42. Park, I. K. et al. Clonal diversification and changes in lipid traits and colony morphology in Mycobacterium abscessus clinical isolates. J. Clin. Microbiol 53, 3438-3447 (2015)

43. Tsai, S. H., Lai, H. C. \& Hu, S. T. Subinhibitory Doses of Aminoglycoside Antibiotics Induce Changes in the Phenotype of Mycobacterium abscessus. Antimicrob. Agents Chemother. 59, 6161-6169 (2015).

44. Cheng, T., Kam, J. Y., Johansen, M. D. \& Oehlers, S. H. High content analysis of granuloma histology and neutrophilic inflammation in adult zebrafish infected with Mycobacterium marinum. Micron 129, 102782 (2020).

45. Bernut, A. et al. Deciphering and imaging pathogenesis and cording of Mycobacterium abscessus in Zebrafish Embryos. J. Vis. Exp., https://doi.org/ $10.3791 / 53130$ (2015).

46. Wu, R. S. et al. A rapid method for directed gene knockout for screening in G0 Zebrafish. Dev. Cell 46, 112-125 (2018). el14.

\section{Acknowledgements}

We thank the Centenary imaging facility core and Sydney Cytometry staff Drs Kristina Jahn, Angela Kurz, and David Liu, for their assistance. We thank Dr Renjing Liu for providing material supplies for this study. Australian National Health and Medical Research Council CJ Martin Early Career Fellowship APP1053407 and Project Grant APP1099912; The University of Sydney Fellowship G197581; NSW Ministry of Health under the NSW Health Early-Mid Career Fellowships Scheme H18/31086; the Kenyon Family Foundation Inflammation Award; Australian-French Association for Research and Innovation (AFRAN) Initiative; The University of Sydney Marie Bashir Institute 2019 Seed Funding to S.H.O. Sydney Medical School Summer Scholarship to J.Y.K. Postdoctoral fellowship granted by Labex EpiGenMed, an "Investissements d'avenir" program ANR-10-LABX-12-01 to M.D.J.; The Fondation pour la Recherche Médicale DEQ20150331719 to L.K.

\section{Author contributions}

J.K. \& K.W.: performed experiments; E.H. \& E.K.: preliminary experiments; S.W., K.L., \& T.C.: C.F.U. recovery assays; P.M.C.: histological analysis; K.K.: provided reagents; J.A.T. \& W.J.B.: supervision of study; M.D.J.: conceived study, supervision of study, wrote manuscript; L.K.: conceived study, provided reagents, supervision of study, wrote manuscript; S.H.O.: conceived study, performed experiments, supervision of study, wrote manuscript.

\section{Competing interests}

The authors declare no competing interests. 


\section{Additional information}

Supplementary information The online version contains supplementary material available at https://doi.org/10.1038/s41467-022-28638-5.

Correspondence and requests for materials should be addressed to Stefan H. Oehlers.

Peer review information Nature Communications thanks Eleanor Marshall and the other, anonymous, reviewers for their contribution to the peer review of this work. Peer reviewer reports are available.

Reprints and permission information is available at http://www.nature.com/reprints

Publisher's note Springer Nature remains neutral with regard to jurisdictional claims in published maps and institutional affiliations. (c) (1) Open Access This article is licensed under a Creative Commons Attribution 4.0 International License, which permits use, sharing, adaptation, distribution and reproduction in any medium or format, as long as you give appropriate credit to the original author(s) and the source, provide a link to the Creative Commons license, and indicate if changes were made. The images or other third party material in this article are included in the article's Creative Commons license, unless indicated otherwise in a credit line to the material. If material is not included in the article's Creative Commons license and your intended use is not permitted by statutory regulation or exceeds the permitted use, you will need to obtain permission directly from the copyright holder. To view a copy of this license, visit http://creativecommons.org/ licenses/by/4.0/.

(C) The Author(s) 2022 